\title{
Computer programs and methodologies for the simulation of DNA sequence data with recombination
}

\author{
Miguel Arenas * \\ Centre for Molecular Biology "Severo Ochoa," Consejo Superior de Investigaciones Científicas, Madrid, Spain
}

Edited by:

Badri Padhukasahasram, Ford, USA

Reviewed by:

Bjørn Østman, Michigan State University, USA

Marcos Perez-Losada, Centro de Investigação em Biodiversidade e Recursos Genéticos, Portugal

${ }^{*}$ Correspondence:

Miguel Arenas, Centre for Molecular Biology "Severo Ochoa," Consejo

Superior de Investigaciones

Científicas - Universidad Autónoma de Madrid, C/Nicolás Cabrera, 1, 28049 Cantoblanco, Madrid, Spain e-mail:marenas@cbm.uam.es
Computer simulations are useful in evolutionary biology for hypothesis testing, to verify analytical methods, to analyze interactions among evolutionary processes, and to estimate evolutionary parameters. In particular, the simulation of DNA sequences with recombination may help in understanding the role of recombination in diverse evolutionary questions, such as the genome structure. Consequently, plenty of computer simulators have been developed to simulate DNA sequence data with recombination. However, the choice of an appropriate tool, among all currently available simulators, is critical if recombination simulations are to be biologically meaningful. This review provides a practical survival guide to commonly used computer programs and methodologies for the simulation of coding and non-coding DNA sequences with recombination. It may help in the correct design of computer simulation experiments of recombination. In addition, the study includes a review of simulation studies investigating the impact of ignoring recombination when performing various evolutionary analyses, such as phylogenetic tree and ancestral sequence reconstructions. Alternative analytical methodologies accounting for recombination are also reviewed.

Keywords: simulation, recombination, recombination breakpoints, recombination hotspots, DNA sequences, recombination phylogenetic bias

\section{INTRODUCTION}

Recombination constitutes a basic and dominant mechanism in molecular evolution, increasing genetic diversity before natural selection operates on the new sequence. Recombination is widespread across nuclear genomes (e.g., Awadalla, 2003; Tsaousis et al., 2005; Fraser et al., 2007; Gaut et al., 2007; Duret and Arndt, 2008) and the importance of its understanding has been long recognized, with crucial implications for genome structure (Reich et al., 2001), phenotypic diversity (Zhang et al., 2002), and genetic diseases (Daly et al., 2001). Moreover, ignoring recombination may bias phylogenetic reconstructions (e.g., Posada, 2001; Posada and Crandall, 2002; Beiko et al., 2008), and the derived inferences (e.g., Schierup and Hein, 2000a; Feil et al., 2001; Anisimova et al., 2003; Arenas and Posada, 2010a,b,c).

The evolutionary importance of recombination (e.g., Robertson et al., 1995; Lukashev, 2005) calls for its accurate detection and measurement (see, Martin et al., 2011). Although some analytical methods have shown an overall better performance than others (Posada and Crandall, 2001; Wiuf et al., 2001), the choice of an appropriate tool also depends on the particular analysis (e.g., detection of recombination breakpoints or estimation of recombination rates), computational costs (some methods are computationally expensive), and the genetic marker. I recommend the following two reviews for helping users to make choices for appropriate methods and computer tools for recombination inference (Posada et al., 2002; Martin et al., 2011).

Computer simulations aim to mimic real world processes. They allow the study of mechanisms that may alter processes or the understanding of complex systems that are analytically intractable
(Peck, 2004). Indeed, the simulation of evolutionary histories is commonly used for hypothesis testing (e.g., Arenas et al., 2008; Pierron et al., 2011), to verify and compare analytical methods or programs (e.g., Westesson and Holmes, 2009; Marttinen et al., 2012), to analyze interactions among evolutionary processes (e.g., Arenas et al., 2012, 2013), or to estimate evolutionary parameters (e.g., Wilson et al., 2009; Beaumont, 2010). Importantly, the choice of an appropriate simulator is critical because simulations should be as realistic as possible in order to mimic a given biological scenario. Although several studies have already reviewed computer simulators in population genetics from global perspectives (e.g., Liu et al., 2008; Arenas, 2012; Arenas and Posada, 2012; Hoban et al., 2012), they have not explored particular methodologies for the simulation of DNA sequences with recombination.

The present study provides an overview of the capabilities of available computer tools and methodologies, and suggests recommendations, for the simulation of DNA sequences with recombination. It also describes some applications of simulated datasets with recombination to show the importance of including recombination in evolutionary analyses. Alternative analytical methodologies that consider recombination are also suggested.

\section{COMPUTER PROGRAMS FOR THE SIMULATION OF DNA DATA UNDER RECOMBINATION}

Recombination can be simulated by the two major simulation approaches commonly used in population genetics, the forward in time (forward-time, where the evolutionary history of an entire population is simulated from the past to the present; e.g., Epperson et al., 2010) and the coalescent (backward-time, which describes a 
backward in time genealogical process from a sample of genes to a single ancestral copy; e.g., Nordborg, 2007; Wakeley, 2008). The forward-time approach can simulate complex processes, including gene-gene interactions and complex selection (e.g., Calafell et al., 2001; Peng et al., 2007), but coalescent simulations are computationally faster and can be recommended for extensive simulation studies (e.g., Beaumont et al., 2002). Table 1 shows an overview of currently available computer programs, for both coalescent and forward-time approaches, to simulate DNA sequences with recombination.

\section{SIMULATION OF CODING DNA SEOUENCES WITH RECOMBINATION}

Direct simulation of coding DNA sequences with recombination can be only performed with a few programs. Using the coalescent approach, the programs Recodon (Arenas and Posada, 2007), CodonRecSim (Anisimova et al., 2003), and NetRecodon (Arenas and Posada, 2010a) allow such simulation, but only the latter program does not force recombination breakpoints to occur between codons, thus allowing more realistic simulations (see Arenas and Posada, 2010a). Concerning the forward-time approach, only the programs GenomePop (Carvajal-Rodriguez, 2008) and SFS_CODE (Hernandez, 2008) implement the simulation of coding sequences with recombination.

Evolutionary scenarios that are not implemented in these programs can be simulated by the following alternative methodology, which is based on the concatenation of two different simulators. First, we simulate an evolutionary history with recombination [an ancestral recombination graph (ARG, see Figure 1A), which contains a tree for each recombinant fragment; Figures 1B-D]. This procedure can be carried out using, for example, the program $m s$ (Hudson, 2002); see also other evolutionary history simulators in (Hoban et al., 2012). Next, we simulate molecular evolution of each coding fragment, according to a user-specified codon-substitution model, along its corresponding simulated tree (further details in Yang, 2006; Fletcher and Yang, 2009). Finally, we just concatenate the simulated coding fragments. The simulation of coding sequence evolution along given trees can be performed, for example, with the program INDELible (Fletcher and Yang, 2009); see also other software in (Arenas, 2012; Arenas and Posada, 2012). The limitation of this methodology is that recombination breakpoints are always assumed to occur between codons and not within codons.

\section{SIMULATION OF NUCLEOTIDE SEQUENCES WITH RECOMBINATION}

A number of computer programs can directly simulate non-coding DNA sequences under recombination (see Table 1). Similarly to the previous subsection, the simulation of non-coding DNA sequences under other evolutionary scenarios, which are not described in the Table 1, can be performed by combining two computer tools. We can use a simulator of recombination evolutionary histories (e.g., ms or msms; Ewing and Hermisson, 2010) followed by a non-coding DNA sequence evolution simulator (e.g., INDELible, Seq-Gen, Rambaut and Grassly, 1997; EVOLVER, Yang, 1997; or indel-Seq-Gen, Strope et al., 2009).

\section{SIMULATION OF GENOMES WITH RECOMBINATION HOTSPOTS}

It is known that the recombination rate is not homogeneous throughout the genome and some regions (hotspot regions) are more likely to suffer recombination (e.g., Gabriel et al., 2002; Zhuang et al., 2002). Consequently, recombination hotspots should be considered for realistic genome simulation.

The simulation of genomes with recombination requires robust and memory-efficient simulators. Programs like fastsimcoal (Excoffier and Foll, 2011) or mlcoalsim (Ramos-Onsins and Mitchell-Olds, 2007) allow for efficient simulations of non-coding genomic regions under recombination (including recombination hotspots). However, these tools do not implement a variety of substitution models (e.g., codon models), or particular evolutionary mechanisms like selection; this may be problematic if we are trying to mimic genome-wide data (see, Arbiza et al., 2011).

Again, an alternative methodology consists of the use of two simulators. A few programs currently implement the simulation of recombination hotspots, namely, SNPsim (Wiuf and Posada, 2003), cosi (Schaffner et al., 2005), GENOME (Liang et al., 2007), mbs (Teshima and Innan, 2009), and msHOT (Hellenthal and Stephens, 2007). Although all these programs simulate particular genetic markers (such as SNPs or STRs), DNA sequence evolution can be simulated upon phylogenetic trees produced by these programs if we use the two-step procedure described above.

\section{SIMULATION OF RECOMBINATION PHYLOGENETIC NETWORKS}

In order to represent a full evolutionary history with recombination, phylogenetic networks should be used instead of forcing the genealogy onto a single tree (Huson and Bryant, 2006). There are two commonly used methodologies for the simulation of recombination networks: direct simulation of the ARG (e.g., Figure 1A) or combining the simulated trees for each recombinant fragment (e.g., Figures 1B-D). To my knowledge, only two programs can really output a simulated ARG, namely, Serial NetEvolve (Buendia and Narasimhan, 2006) and NetRecodon (Arenas and Posada, 2010a), where the ARG can be visualized and analyzed using the NetTest web server (Arenas et al., 2010) ${ }^{1}$. On the other hand, trees can be combined to generate a network using tools like CombineTrees (see for a review, Woolley et al., 2008) ${ }^{2}$.

\section{RECOMBINATION SIMULATION FOR ANALYZING THE INFLUENCE OF RECOMBINATION ON PHYLOGENETIC INFERENCES}

This section outlines three computer simulation studies where ignoring recombination leads to biased phylogenetic inferences. Alternative phylogenetic inference methodologies considering recombination are also suggested.

\section{INFLUENCE OF RECOMBINATION ON PHYLOGENETIC TREE RECONSTRUCTION}

Schierup and Hein (2000a) simulated samples under the coalescent with recombination (Hudson, 1983). Then, from the simulated genealogy, they simulated nucleotide sequence evolution under the Jukes-Cantor (JC) and Kimura's two-parameter (K2P) nucleotide substitution models of evolution. The simulated datasets were analyzed using programs for phylogenetic tree

\footnotetext{
${ }^{1}$ http://darwin.uvigo.es/software/nettest/

${ }^{2}$ http://applications.lanevol.org/combineTrees/
} 


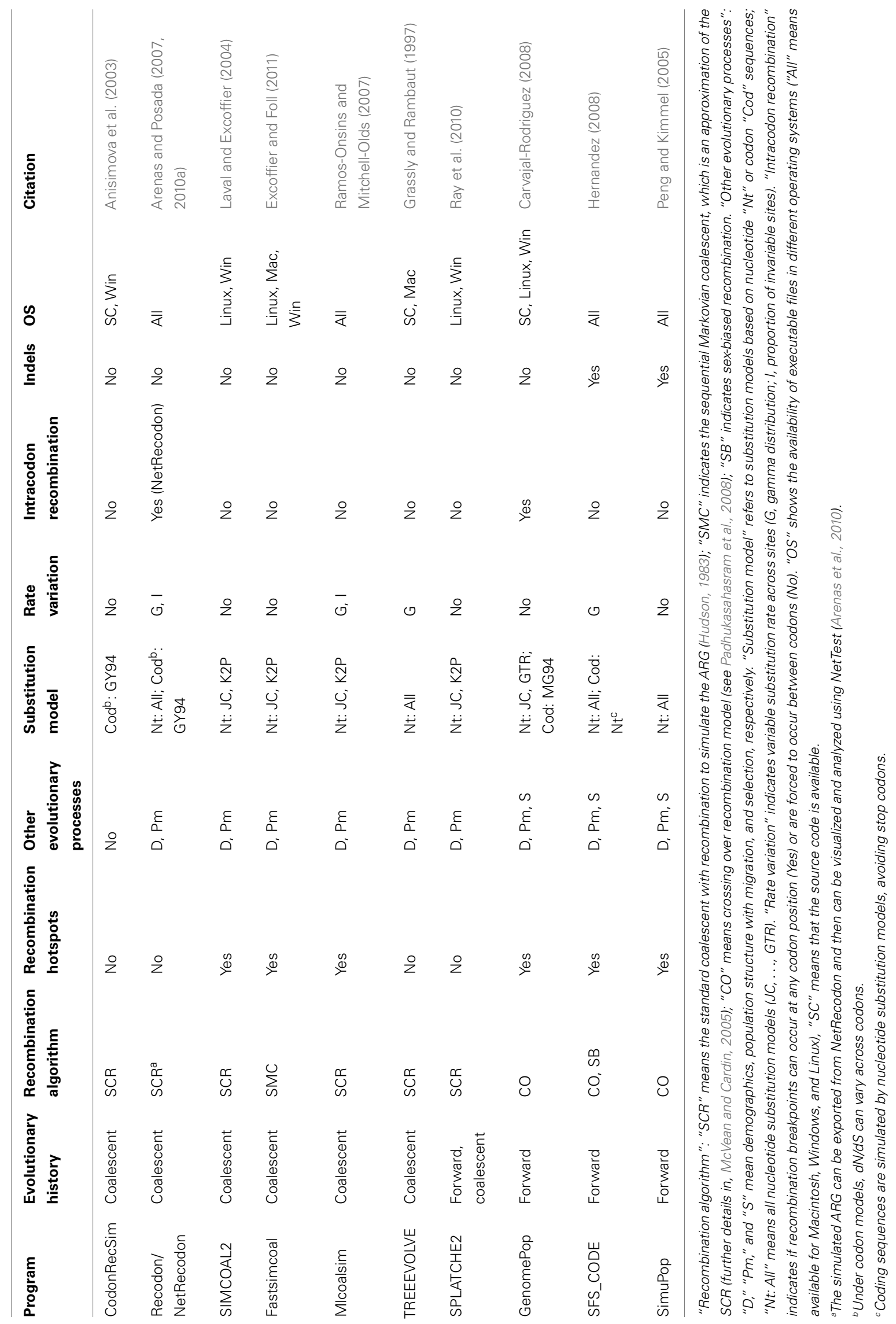



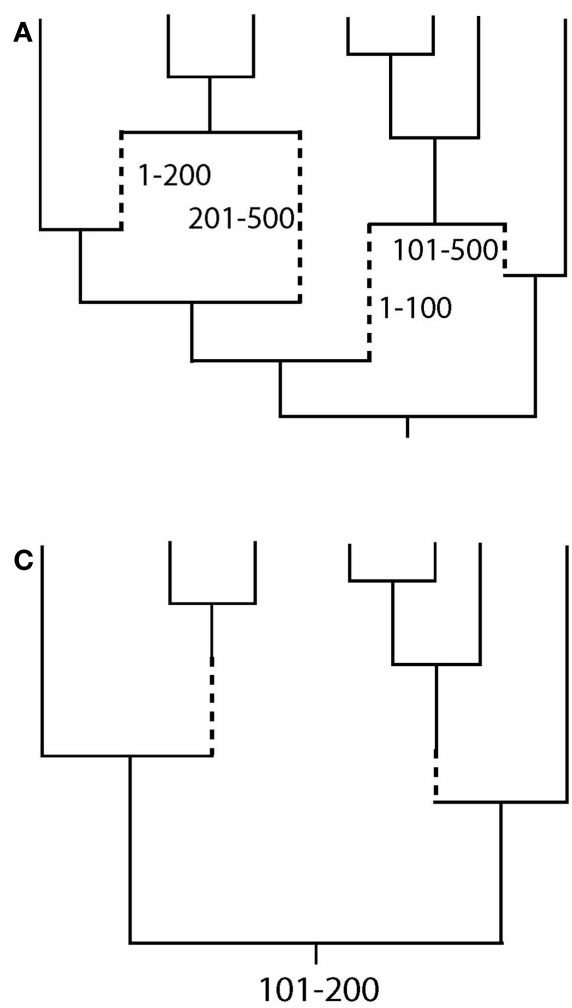

FIGURE 1 | Example of an ancestral recombination graph (ARG) with the corresponding embedded trees for each recombinant fragment. (A) ARG based on two recombination events with breakpoints at positions 100 and 200. Dashed lines indicate branches for recombinant fragments. (B-D)

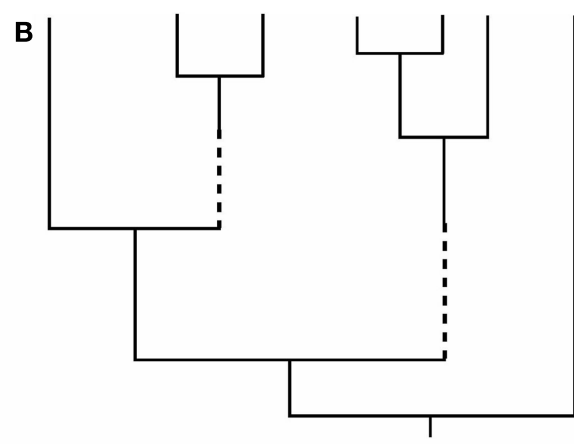

$1-100$

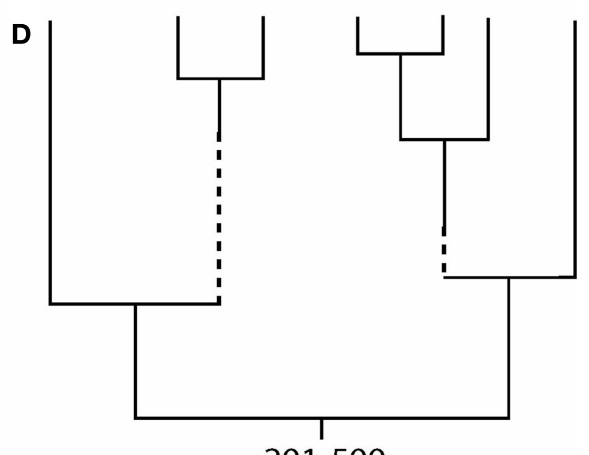

$201-500$

Embedded tree for each recombinant fragment. Note that topologies and branch lengths may differ across trees. Finally, the simulation of sequence evolution can be performed site by site along the corresponding tree (see, Yang, 2006; Fletcher and Yang, 2009). reconstruction by both distance-based methods and maximumlikelihood (ML) methods. Ignoring recombination biased the inferred phylogenetic trees toward larger terminal branches, smaller times to the most recent common ancestor (MRCA) and incorrect topologies (Schierup and Hein, 2000a). In addition, ignoring recombination led to overestimation of the substitution rate heterogeneity, apparent homoplasies and loss of molecular clock (Schierup and Hein, 2000a,b). Later, Posada (2001) analyzed the molecular clock hypothesis on four empirical datasets. In particular, the author applied a triplet likelihood ratio test (test for equality of evolutionary rates among three species, called a relative-rate test, RRT), which is independent of topology and might be unbiased by recombination. Results showed that recombinant data did not allow a good fit to the molecular clock when using classical likelihood ratio tests (LRT). However, the molecular clock was not rejected when using the RRT test. Thus, this test could be recommended for testing a molecular clock in the presence of recombination. In addition, phylogenetic incongruence in empirical data was also observed as a consequence of ignoring recombination (e.g., Worobey and Holmes, 1999; Feil et al., 2001).

These findings, consequently, suggest biases in derived evolutionary analyses based on phylogenetic reconstructions that ignore recombination. As an alternative, there are two methodologies of phylogenetic reconstruction accounting for recombination:
- Inference of a single phylogenetic network (e.g., Figure 1A; Griffiths and Marjoram, 1997; Huson and Bryant, 2006). Recombination networks can be inferred by using computer programs like SplitsTree (Huson, 1998; Huson and Bryant, 2006).

- Inference of a set of phylogenetic trees, where each tree corresponds to the evolutionary history of each recombinant fragment (e.g., Figures 1B-D). The methodology consists of the detection of recombination breakpoints (see for a review, Martin et al., 2011) followed by a phylogenetic tree reconstruction for each recombinant fragment.

Both methodologies correctly account for recombination and the choice should be based on the posterior application. For example, the phylogenetic network may help for an easy visualization of clades and phylogenetic relationships (e.g., Maughan and Redfield, 2009). By contrast, the simulation of sequence evolution requires a phylogenetic tree for each recombinant fragment (e.g., Fletcher and Yang, 2009).

\section{INFLUENCE OF RECOMBINATION ON ANCESTRAL SEQUENCE RECONSTRUCTION}

Recently, Arenas and Posada (2010c) analyzed the effect of considering recombination on ancestral sequence reconstruction (ASR). They performed extensive simulations of nucleotide, codon, and amino acid data by using the coalescent with recombination 
approach implemented in NetRecodon. They then reconstructed ancestral sequences with different ASR methods (joint ML, marginal ML, and empirical Bayes). Results clearly indicated that ignoring recombination biases the reconstruction of ancestral sequences, regardless of the method or software used. This ASR error can be partially reduced if recombination is considered (Arenas and Posada, 2010c). The methodology consists of four steps: the detection of recombination breakpoints, the reconstruction of a phylogenetic tree for each recombinant fragment, the reconstruction of ancestral fragments by using the corresponding trees and, finally, the concatenation of the ancestral fragments to generate the entire ancestral sequence. The Datamonkey web server (Kosakovsky Pond and Frost, 2005) ${ }^{3}$ and the Hyphy package (Kosakovsky Pond et al., 2005) have automated the whole procedure described above to infer ancestral sequences with consideration of recombination.

Arenas and Posada (2010c) also analyzed empirical data, in particular two datasets of the env region of HIV-1. They inferred ancestral sequences both ignoring and considering recombination, using the methodology described above, and observed a different number of CTL epitopes depending on whether recombination was considered or not.

\section{INFLUENCE OF RECOMBINATION ON THE DETECTION OF MOLECULAR ADAPTATION}

The detection of molecular adaptation (based on the nonsynonymous/synonymous substitution rate ratio, hereafter $\mathrm{dN} / \mathrm{dS}$ ) is commonly used at both global (entire sequence) and local (codon) levels. Indeed, these analyses have commonly been applied to datasets collected from highly recombinant viruses and bacteria (e.g., Perez-Losada et al., 2009, 2011; Bozek and Lengauer, 2010). Several studies have shown the impact of recombination on the estimation of dN/dS (e.g., Anisimova et al., 2003; Arenas and Posada, 2010a). After simulating coding data under a variety of codon-substitution models for heterogeneous selection pressure (see, Yang et al., 2000) and different levels of recombination, they selected those heterogeneous codon models that best fitted the simulated data by using LRTs. Results showed a weak impact of recombination on the estimation of global $\mathrm{dN} / \mathrm{dS}$ but a strong effect on the estimation of local $\mathrm{dN} / \mathrm{dS}$, in particular by increasing the number of false-positively selected sites (PSS). An alternative methodology to reduce these errors consists of the detection of recombination breakpoints followed by the reconstruction of a phylogenetic tree for each recombinant fragment and, finally, the estimation of $\mathrm{dN} / \mathrm{dS}$ by using the corresponding trees (see, Kosakovsky Pond et al., 2006). This methodology was applied in (Perez-Losada et al., 2009, 2011). Again, the Datamonkey web server and the Hyphy package have automated this whole procedure to estimate $\mathrm{dN} / \mathrm{dS}$ while accounting for recombination.

Recombination might also affect other evolutionary inferences. For example, it could bias those analytical methods based on the coalescent without recombination (e.g., BEAST; Drummond and Rambaut, 2007). However these influences have not yet been rigorously evaluated.

${ }^{3}$ http://www.datamonkey.org/
Another interesting question concerns the influence of recombination on genetic diversity. Spencer et al. (2006) studied this in humans and found that recombination only affects genetic diversity at recombination hotspots. However, such hotspots did not alter substitution rates, perhaps because recombination rates were always low. By contrast, large recombination rates (common in a variety of viruses and bacteria) may strongly increase genetic diversity and bring novel lineages (e.g., He et al., 2010).

At this point, I would suggest the approximate Bayesian computation (ABC) approach (see for a review, Beaumont, 2010) to estimate evolutionary parameters accounting for recombination. $\mathrm{ABC}$ is based on computer simulations and provides an alternative for those analyses where the likelihood function cannot be computed. Simulations can be performed according to a prior distribution for recombination rate (among other parameters) and then, by a rejection or a regression method, a posterior distribution can be computed to obtain the parameter estimates (Beaumont et al., 2002). For example, Wilson et al. (2009) applied ABC for joint estimation of a set of evolutionary parameters, such as substitution rate, $\mathrm{dN} / \mathrm{dS}$ and recombination rate. By this methodology, the influence of recombination on other evolutionary mechanisms is accounted for, but only if it is indeed implemented in the computer simulator.

\section{CONCLUSION}

This review provides a practical guide to the state of the art in software, and recommends methodologies, for simulating coding and non-coding sequence data with recombination, including recombination hotspots. Currently, only three programs implement the direct simulation of coding data with recombination. These programs will not cover every evolutionary scenario, but this problem can be circumvented by the use of two simulators, one for the evolutionary history and another for sequence evolution. It is also important to consider intracodon recombination (Arenas and Posada, 2010a), because $2 / 3$ of recombination events are expected to occur within codons. By contrast, the simulation of non-coding sequences with recombination can be performed by a variety of programs. Here again, two simulators may be combined where necessary.

Among many other applications (e.g., Sun et al., 2011; Marttinen et al., 2012), the simulation of DNA data with recombination has been especially important for demonstrating the strong influence of recombination on phylogenetic tree reconstruction and derived analyses, such as ASR or dN/dS estimation. However, some alternative methodologies have been developed for phylogenetic inference accounting for recombination.

The current set of computer tools to simulate DNA sequences with recombination can cover a wide range of evolutionary scenarios. However, some scenarios are still difficult to simulate and will require the development of more complex simulators. For example, next-generation sequencing (NGS) technologies now deliver fast and accurate genome sequences (Metzker, 2010) that may call for simulations of entire genomes accounting for recombination (including recombination hotspots; e.g., Westesson and Holmes, 2009; Marttinen et al., 2012), as well as other evolutionary 
mechanisms like natural selection. Indeed, the simulation of DNA evolution should be performed by using different substitution models for each genomic region (Arbiza et al., 2011). Moreover, I would expect interactions between the different evolutionary forces, such as joint influences of natural selection and recombination on dN/dS (e.g., Anisimova et al., 2003; Kryazhimskiy and Plotkin, 2008) or of structural protein energies on sequence evolution (e.g., Bastolla et al., 2007; Arenas et al., 2009; Grahnen et al., 2011). To my knowledge, there is currently no tool to simulate sequences accounting for all these evolutionary features, including interactions among them. On the other hand, there is also a demand for fast simulations, in particular for applying $A B C$ or Bayesian model-choice approaches that require extensive simulations (see recombination examples in, Wilson et al., 2009; Nunes and Balding, 2010; Sohn et al., 2012).

\section{REFERENCES}

Anisimova, M., Nielsen, R., and Yang, Z. (2003). Effect of recombination on the accuracy of the likelihood method for detecting positive selection at amino acid sites. Genetics 164, 1229-1236.

Arbiza, L., Patricio, M., Dopazo, H., and Posada, D. (2011). Genome-wide heterogeneity of nucleotide substitution model fit. Genome Biol. Evol. 3, 896-908.

Arenas, M. (2012). Simulation of molecular data under diverse evolutionary scenarios. PLoS Comput. Biol. 8:e1002495. doi:10.1371/journal.pcbi.1002495

Arenas, M., Francois, O., Currat, M., Ray, N., and Excoffier, L. (2013). Influence of admixture and paleolithic range contractions on current European diversity gradients. Mol. Biol. Evol. 30, 57-61.

Arenas, M., Patricio, M., Posada, D., and Valiente, G. (2010). Characterization of phylogenetic networks with nettest. BMC Bioinformatics 11:268. doi:10.1186/1471-2105-11-268

Arenas, M., and Posada, D. (2007). Recodon: coalescent simulation of coding DNA sequences with recombination, migration and demography. BMC Bioinformatics 8:458. doi:10.1186/1471-2105-8-458

Arenas, M., and Posada, D. (2010a). Coalescent simulation of intracodon recombination. Genetics 184, 429-437.

Arenas, M., and Posada, D. (2010b). Computational design of centralized HIV-1 genes. Curr. HIV Res. 8, 613-621.

Arenas, M., and Posada, D. (2010c). The effect of recombination on the reconstruction of ancestral sequences. Genetics 184, 1133-1139.

Arenas, M., and Posada, D. (2012). "Simulation of coding sequence evolution," in Codon Evolution, eds G.

In conclusion, there is a need to innovate continuously in fast and complex simulators of DNA sequences with recombination and I expect future advances in this area.

\section{ACKNOWLEDGMENTS}

I want to thank Badri Padhukasahasram, Guest Associate Editor of Frontiers in Evolutionary and Population Genetics, for the invitation to contribute with this review to the Research Topic "Inference of recombination and gene-conversion from whole genome sequence variation data." Indeed, I also want to thank the Journal Frontiers in Evolutionary and Population Genetics for a waiver to cover publication costs. I thank Dr Richard M. Gunton for helpful comments. I thank two reviewers for insightful comments and suggestions. I thank the Spanish Government for the "Juan de la Cierva" fellowship, JCI-2011-10452.

recombinant network. Bioinformatics 22, 2313-2314.

Calafell, F., Grigorenko, E. L., Chikanian, A. A., and Kidd, K. K. (2001). Haplotype evolution and linkage disequilibrium: a simulation study. Hum. Hered. 51, 85-96.

Carvajal-Rodriguez, A. (2008). GENOMEPOP: a program to simulate genomes in populations. BMC Bioinformatics 9:223. doi:10.1186/1471-2105-9-223

Daly, M. J., Rioux, J. D., Schaffner, S. F., Hudson, T. J., and Lander, E. S. (2001). High-resolution haplotype structure in the human genome. Nat. Genet. 29, 229-232.

Drummond, A. J., and Rambaut, A. (2007). BEAST: Bayesian evolutionary analysis by sampling trees. $B M C$ Evol. Biol. 7:214. doi:10.1186/14712148-7-214

Duret, L., and Arndt, P. F. (2008). The impact of recombination on nucleotide substitutions in the human genome. PLoS Genet. 4:e1000071. doi:10.1371/journal.pgen.1000071

Epperson, B. K., McRae, B. H., Scribner, K., Cushman, S. A., Rosenberg, M. S., Fortin, M. J., et al. (2010). Utility of computer simulations in landscape genetics. Mol. Ecol. 19, 3549-3564.

Ewing, G., and Hermisson, J. (2010). MSMS: a coalescent simulation program including recombination, demographic structure and selection at a single locus. Bioinformatics 26, 2064-2065.

Excoffier, L., and Foll, M. (2011). Fastsimcoal: a continuous-time coalescent simulator of genomic diversity under arbitrarily complex evolutionary scenarios. Bioinformatics 27 , 1332-1334.

Feil, E. J., Holmes, E. C., Bessen, D. E., Chan, M.-S., Day, N. P. J., Enright, M. C., et al. (2001). Recombination within natural populations of pathogenic bacteria: Short-term empirical estimates and long-term phylogenetic consequences. Proc. Natl. Acad. Sci. U.S.A. 98, 182-187.

Fletcher, W., and Yang, Z. (2009). INDELible: a flexible simulator of biological sequence evolution. Mol. Biol. Evol. 26, 1879-1888.

Fraser, C., Hanage, W. P., and Spratt, B. G. (2007). Recombination and the nature of bacterial speciation. Science 315, 476-480.

Gabriel, S. B., Schaffner, S. F., Nguyen, H., Moore, J. M., Roy, J., Blumenstiel, B., et al. (2002). The structure of haplotype blocks in the human genome. Science 296, 2225-2229.

Gaut, B. S., Wright, S. I., Rizzon, C., Dvorak, J., and Anderson, L. K. (2007). Recombination: an underappreciated factor in the evolution of plant genomes. Nat. Rev. Genet. 8 , 77-84.

Grahnen, J. A., Nandakumar, P., Kubelka, J., and Liberles, D. A. (2011). Biophysical and structural considerations for protein sequence evolution. BMC Evol. Biol. 11:361. doi:10.1186/1471-2148-11-361

Grassly, N. C., and Rambaut, A. (1997). Treevolve: A Program to Simulate the Evolution of DNA Sequences Under Different Population Dynamic Scenarios. Oxford: Department of Zoology, Wellcome Centre for Infectious Disease, Oxford University.

Griffiths, R. C., and Marjoram, P. (1997). "An ancestral recombination graph," in Progress in Popula tion Genetics and Human Evolution, eds P. Donelly and S. Tavaré (Berlin: Springer-Verlag), 257-270.

He, C. Q., Ding, N. Z., He, M., Li, S. N., Wang, X. M., He, H. B., et al. (2010). Intragenic recombination as a mechanism of genetic diversity in bluetongue virus. J. Virol. 84, 11487-11495. 
Hellenthal, G., and Stephens, M. (2007). msHOT: modifying Hudson's ms simulator to incorporate crossover and gene conversion hotspots. Bioinformatics $23,520-521$.

Hernandez, R. D. (2008). A flexible forward simulator for populations subject to selection and demography. Bioinformatics 24, 2786-2787.

Hoban, S., Bertorelle, G., and Gaggiotti, O. E. (2012). Computer simulations: tools for population and evolutionary genetics. Nat. Rev. Genet. 13, 110-122.

Hudson, R. R. (1983). Properties of a neutral allele model with intragenic recombination. Theor. Popul. Biol. $23,183-201$.

Hudson, R. R. (2002). Generating samples under a Wright-Fisher neutral model of genetic variation. Bioinformatics 18, 337-338.

Huson, D. H. (1998). Splitstree: analyzing and visualizing evolutionary data. Bioinformatics 14, 68-73.

Huson, D. H., and Bryant, D. (2006). Application of phylogenetic networks in evolutionary studies. Mol. Biol. Evol. 23, 254-267.

Kosakovsky Pond, S. L., and Frost, S. D. (2005). Datamonkey: rapid detection of selective pressure on individual sites of codon alignments. Bioinformatics 21, 2531-2533.

Kosakovsky Pond, S. L., Frost, S. D., and Muse, S. V. (2005). HYPHY: hypothesis testing using phylogenies. Bioinformatics 21, 676-679.

Kosakovsky Pond, S. L., Posada, D., Gravenor, M. B., Woelk, C. H., and Frost, S. D. (2006). Automated phylogenetic detection of recombination using a genetic algorithm. Mol. Biol. Evol. 23, 1891-1901.

Kryazhimskiy, S., and Plotkin, J. B. (2008). The population genetics of dN/dS. PLoS Genet. 4:e1000304. doi:10.1371/journal.pgen.1000304

Laval, G., and Excoffier, L. (2004). SIMCOAL 2.0: a program to simulate genomic diversity over large recombining regions in a subdivided population with a complex history. Bioinformatics 20, 2485-2487.

Liang, L., Zollner, S., and Abecasis, G. R. (2007). GENOME: a rapid coalescent-based whole genome simulator. Bioinformatics 23, 1565-1567.

Liu, Y., Athanasiadis, G., and Weale, M. E. (2008). A survey of genetic simulation software for population and epidemiological studies. Hum. Genomics 3, 79-86.

Lukashev, A. N. (2005). Role of recombination in evolution of enteroviruses. Rev. Med. Virol. 15, 157-167.
Martin, D. P., Lemey, P., and Posada, D. (2011). Analysing recombination in nucleotide sequences. Mol. Ecol. Resour. 11, 943-955.

Marttinen, P., Hanage, W. P., Croucher, N. J., Connor, T. R., Harris, S. R., Bentley, S. D., et al. (2012). Detection of recombination events in bacterial genomes from large population samples. Nucleic Acids Res. 40, e6.

Maughan, H., and Redfield, R. J. (2009). Tracing the evolution of competence in Haemophilus influenzae. PLoS ONE 4:e5854. doi:10.1371/journal.pone.0005854

McVean, G. A., and Cardin, N. J. (2005). Approximating the coalescent with recombination. Philos. Trans. R. Soc. Lond. B Biol. Sci. 360, 1387-1393.

Metzker, M. L. (2010). Sequencing technologies - the next generation. Nat. Rev. Genet. 11, 31-46.

Nordborg, M. (2007). "Coalescent theory," in Handbook of Statistical Genetics, 3rd Edn, eds D. J. Balding, M. Bishop, and C. Cannings (Chichester: John Wiley \& Sons Ltd), 843-877.

Nunes, M. A., and Balding, D. J. (2010). On optimal selection of summary statistics for approximate Bayesian computation. Stat. Appl. Genet. Mol. Biol. 9, 34.

Padhukasahasram, B., Marjoram, P., Wall, J. D., Bustamante, C. D., and Nordborg, M. (2008). Exploring population genetic models with recombination using efficient forward-time simulations. Genetics 178, 2417-2427.

Peck, S. L. (2004). Simulation as experiment: a philosophical reassessment for biological modeling. Trends Ecol. Evol. (Amst.) 19, 530-534.

Peng, B., Amos, C. I., and Kimmel, M. (2007). Forward-time simulations of human populations with complex diseases. PLoS Genet. 3:e47. doi:10.1371/journal.pgen.0030047

Peng, B., and Kimmel, M. (2005). Simupop: a forward-time population genetics simulation environment. Bioinformatics 21, 3686-3687.

Perez-Losada, M., Jobes, D. V., Sinangil, F., Crandall, K. A., Arenas, M. Posada, D., et al. (2011). Phylodynamics of HIV-1 from a phase III AIDS vaccine trial in Bangkok, Thailand. PLoS ONE 6:e16902. doi:10.1371/journal.pone.0016902

Perez-Losada, M., Posada, D., Arenas, M., Jobes, D. V., Sinangil, F., Berman, P. W., et al. (2009). Ethnic differences in the adaptation rate of HIV gp120 from a vaccine trial. Retrovirology 6 , 67.

Pierron, D., Chang, I., Arachiche, A., Heiske, M., Thomas, O., Borlin, M., et al. (2011). Mutation rate switch inside Eurasian mitochondrial haplogroups: impact of selection and consequences for dating settlement in Europe. PLoS ONE 6:e21543. doi:10.1371/journal.pone.0021543

Posada, D. (2001). Unveiling the molecular clock in the presence of recombination. Mol. Biol. Evol. 18 1976-1978.

Posada, D., and Crandall, K. A. (2001). Evaluation of methods for detecting recombination from DNA sequences: computer simulations. Proc. Natl. Acad. Sci. U.S.A. 98 13757-13762.

Posada, D., and Crandall, K. A. (2002) The effect of recombination on the accuracy of phylogeny estimation. $J$. Mol. Evol. 54, 396-402.

Posada, D., Crandall, K. A., and Holmes, E. C. (2002). Recombination in evolutionary genomics. Annu. Rev Genet. 36, 75-97.

Rambaut, A., and Grassly, N. C. (1997) Seq-gen: an application for the Monte carlo simulation of DNA sequence evolution along phylogenetic trees. Comput. Appl. Biosci. 13 , 235-238.

Ramos-Onsins, S. E., and MitchellOlds, T. (2007). Mlcoalsim: multilocus coalescent simulations. Evol. Bioinform. Online 3, 41-44.

Ray, N., Currat, M., Foll, M., and Excoffier, L. (2010). SPLATCHE2: a spatially explicit simulation framework for complex demography, genetic admixture and recombination. Bioinformatics 26, 2993-2994.

Reich, D. E., Cargill, M., Bolk, S., Ireland, J., Sabeti, P. C., Richter, D. J., et al. (2001). Linkage disequilibrium in the human genome. Nature 411, 199-204.

Robertson, D. L., Sharp, P. M., McCutchan, F. E., and Hahn, B. H. (1995). Recombination in HIV-1. Nature 374, 124-126.

Schaffner, S. F., Foo, C., Gabriel, S., Reich, D., Daly, M. J., and Altshuler, D. (2005). Calibrating a coalescent simulation of human genome sequence variation. Genome Res. 15 , 1576-1583.

Schierup, M. H., and Hein, J. (2000a). Consequences of recombination on traditional phylogenetic analysis. Genetics 156, 879-891.

Schierup, M. H., and Hein, J. (2000b). Recombination and the molecular clock. Mol. Biol. Evol. 17, 1578-1579.

Sohn, K. A., Ghahramani, Z., and Xing, E. P. (2012). Robust estimation of local genetic ancestry in admixed populations using a nonparametric Bayesian approach. Genetics 191, 1295-1308.
Spencer, C. C., Deloukas, P., Hunt, S., Mullikin, J., Myers, S., Silverman, B., et al. (2006). The influence of recombination on human genetic diversity. PLoS Genet. 2:e148. doi:10.1371/journal.pgen.0020148

Strope, C. L., Abel, K., Scott, S. D., and Moriyama, E. N. (2009). Biological sequence simulation for testing complex evolutionary hypotheses: indel-seq-gen version 2.0. Mol. Biol. Evol. 26, 2581-2593.

Sun, S., Evans, B. J., and Golding, G. B. (2011). "Patchy-tachy" leads to false positives for recombination. Mol. Biol. Evol. 28, 2549-2559.

Teshima, K. M., and Innan, H. (2009). Mbs: modifying Hudson's ms software to generate samples of DNA sequences with a biallelic site under selection. BMC Bioinformatics 10:166. doi:10.1186/1471-210510-166

Tsaousis, A. D., Martin, D. P., Ladoukakis, E. D., Posada, D., and Zouros, E. (2005). Widespread Recombination in Published Animal mtDNA Sequences. Mol. Biol. Evol. 22, 925-933.

Wakeley, J. (2008). Coalescent Theory: An Introduction. Greenwood Village: Roberts and Company Publishers.

Westesson, O., and Holmes, I. (2009). Accurate detection of recombinant breakpoints in whole-genome alignments. PLoS Comput. Biol. 5:e1000318. doi:10.1371/journal. pcbi. 1000318

Wilson, D. J., Gabriel, E., Leatherbarrow, A. J., Cheesbrough, J., Gee, S., Bolton, E., et al. (2009). Rapid evolution and the importance of recombination to the gastroenteric pathogen Campylobacter jejuni. Mol. Biol. Evol. 26, 385-397.

Wiuf, C., Christensen, T., and Hein, J. (2001). A simulation study of the reliability of recombination detection methods. Mol. Biol. Evol. 18, 1929-1939.

Wiuf, C., and Posada, D. (2003). A coalescent model of recombination hotspots. Genetics 164 407-417.

Woolley, S. M., Posada, D., and Crandall, K. A. (2008). A comparison of phylogenetic network methods using computer simulation. PLoS ONE 3:e1913. doi:10.1371/journal.pone.0001913

Worobey, M., and Holmes, E. C. (1999). Evolutionary aspects of recombination in RNA viruses. J. Gen. Virol. 80, 2535-2543.

Yang, Z. (1997). PAML: a program package for phylogenetic analysis by maximum likelihood. Comput. Appl. Biosci. 13, 555-556. 
Yang, Z. (2006). Computational Molecular Evolution. Oxford: Oxford University Press.

Yang, Z., Nielsen, R., Goldman, N., and Pedersen, A.-M. K. (2000). Codonsubstitution models for heterogeneous selection pressure at amino acid sites. Genetics 155, 431-449.

Zhang, Y. X., Perry, K., Vinci, V. A., Powell, K., Stemmer, W. P., and del Cardayre, S. B. (2002). Genome shuffling leads to rapid phenotypic improvement in bacteria. Nature 415, 644-646.

Zhuang, J., Jetzt, A. E., Sun, G., Yu, H., Klarmann, G., Ron, Y., et al. (2002). Human immunodeficiency virus type 1 recombination: rate, fidelity, and putative hot spots. J. Virol. 76, 11273-11282.

Conflict of Interest Statement: The author declares that the research was conducted in the absence of any commercial or financial relationships that could be construed as a potential conflict of interest.

Received: 20 November 2012; accepted: 17 January 2013; published online: 01 February 2013.

Citation: Arenas M (2013) Computer programs and methodologies for the simulation of DNA sequence data with recombination. Front. Gene. 4:9. doi 10.3389/fgene.2013.00009
This article was submitted to Frontiers in Evolutionary and Population Genetics, a specialty of Frontiers in Genetics.

Copyright (c) 2013 Arenas. This is an open-access article distributed under the terms of the Creative Commons Attribution License, which permits use, distribution and reproduction in other forums, provided the original authors and source are credited and subject to any copyright notices concerning any third-party graphics etc. 